\title{
Enhancements to Linear Least Squares Localization Through Reference Selection and ML Estimation
}

\author{
Ismail Guvenc $^{1}$, Sinan Gezici ${ }^{2}$, Fujio Watanabe ${ }^{1}$, and Hiroshi Inamura ${ }^{1}$ \\ ${ }^{1}$ DoCoMo Communications Laboratories USA, Inc., 3240 Hillview Avenue, Palo Alto, CA, 94304, USA \\ ${ }^{2}$ Department of Electrical and Electronics Engineering, Bilkent University, Bilkent, Ankara, 06800, Turkey \\ Email: \{iguvenc, watanabe, inamura\}@docomolabs-usa.com, gezici@ee.bilkent.edu.tr
}

\begin{abstract}
Linear least squares (LLS) estimation is a low complexity but sub-optimum method for estimating the location of a mobile terminal (MT) from some distance measurements. It requires selecting one of the fixed terminals (FTs) as a reference FT for obtaining a linear set of expressions. However, selection of the reference FT is commonly performed arbitrarily in the literature. In this paper, a method for selection of the reference FT is proposed, which improves the location accuracy compared to a fixed selection of the reference FT. Moreover, a covariancematrix based LLS estimator is proposed in line of sight (LOS) and non-LOS (NLOS) environments which further improves accuracy since the correlations between the observations are exploited. Simulation results prove the effectiveness of the proposed techniques.
\end{abstract}

Index Terms - Least-Squares (LS) Estimation, Linearization, Maximum Likelihood Estimation (MLE), Reference Selection, Wireless Localization.

\section{INTRODUCTION}

Accurate location estimation is a challenging and important problem in today's wireless systems such as the cellular networks, wireless local area networks, and wireless sensor networks [1], [2]. These systems may employ numerous techniques to solve for the position of a mobile terminal (MT) from a set of measured distances. If the variance of distance measurements at each of the MTs is available, it is well known that the maximum likelihood (ML) solution can be obtained using a weighed non-linear least squares (WNLS) approach [1]. If no information about the measured distance variances is available, or if they are assumed identical, a non-linear least squares (NLS) solution can be obtained by using uniform weighting. Solving the NLS problem requires an explicit minimization of a loss function, and hence necessitates numerical search methods such as the steepest descent or the Gauss-Newton techniques. Such techniques may be computationally costly and they typically require good initialization in order to avoid converging to the local minima of the loss function [3].

In order to obtain a closed form solution and avoid explicit minimization of the loss function, the set of expressions corresponding to each of the observations can be linearized using the Taylor series expansion [4]. However, such an approach still requires an intermediate location estimate to obtain the Jacobian matrix, which should be sufficiently close to the true location of the MT for the linearity assumption to hold.

An alternative linear least squares (LLS) solution based on the measured distances was initially proposed in [5]. It selects one of the fixed terminals (FTs) as a reference, and subtracts the expressions corresponding to this reference FT from the other $(N-1)$ expressions in order to cancel the nonlinear terms, where $N$ denotes the number of observations. Eventually, once a linear set of expressions are obtained, a simple LS matrix solution yields the location of the MT. Some different versions of this LLS solution were also presented later in the literature. For example, in [6], multiple sets of linear expressions are obtained, where for each set a different FT is selected as the reference. This provides $N \times(N-1) / 2$ total number of different equations, likely yielding a better location estimate compared to a random selection of the reference FT. In another work [7], a different averaging technique is proposed. First, the non-linear expressions are averaged over all the FTs. Then, the resulting expression is subtracted from the rest of the expressions which still cancels out the nonlinear terms. In [8], the cost functions for LLS and NLS were compared through simulations, which shows that NLS usually performs better than the LLS in most of the topologies. A similar result was also observed in [7], which clearly shows the sub-optimality of the LLS for position estimation. Theoretical mean square errors (MSEs) of the LLS for the line-of-sight (LOS) and non-LOS (NLOS) scenarios were derived in [9].

While LLS is a sub-optimum location estimation technique, with a reasonable position estimation accuracy, it can be used by itself to obtain the MT location due to its lower implementation complexity compared to other iterative techniques (such as the NLS). Moreover, in other high-accuracy techniques (including the NLS approach and linearization based on the Taylor series), it can be used to obtain an initial location estimate for initializing the high-accuracy location algorithm (see e.g. [10]). A good initialization may considerably decrease the computational complexity and eventual localization error of a high-accuracy technique. Therefore, improving the accuracy of the LLS localization technique carries importance from multiple perspectives.

In this paper, we propose two different techniques for improving the performances of the LLS techniques in [5][7]. First, a simple yet effective method for selecting the reference FT for linearization is proposed. Second, the covariance matrices of the observations for different scenarios are derived, and maximum likelihood estimators (MLEs) for these scenarios are introduced. Simulation results in line-ofsight (LOS) and non-LOS (NLOS) scenarios show that the proposed techniques yield better accuracies compared to priorart techniques. The paper is organized as follows. Section II introduces the system model and briefly reviews the prior art techniques. The proposed LLS techniques are introduced in 
This full text paper was peer reviewed at the direction of IEEE Communications Society subject matter experts for publication in the WCNC 2008 proceedings.

Section III. Section IV provides the simulation results and the last section concludes the paper.

\section{SySTEM MODEL}

Consider a wireless network as in Fig. 1 where there are $N$ FTs, $\mathbf{x}=\left[\begin{array}{ll}x & y\end{array}\right]^{T}$ is the estimate of the MT location, $\mathbf{x}_{i}=\left[\begin{array}{ll}x_{i} & y_{i}\end{array}\right]^{T}$ is the position of the $i$ th FT, $\hat{d}_{i}$ is the measured distance between the MT and the $i$ th FT commonly modeled as

$$
\hat{d}_{i}=d_{i}+b_{i}+n_{i}=c \tau_{i}, \quad i=1,2, \ldots, N
$$

where $\tau_{i}$ is the TOA of the signal at the $i$ th FT, $d_{i}$ is the actual distance between the MT and the $i$ th FT, $n_{i} \sim \mathcal{N}\left(0, \sigma_{i}^{2}\right)$ is the additive white Gaussian noise (AWGN) with variance $\sigma_{i}^{2}$, and $b_{i}$ is a positive distance bias introduced due to LOS blockage, which is zero for LOS FTs. For the rest of the paper, we assume that the measurement variance is the same for all the FTs (i.e., $\sigma_{i}^{2}=\sigma^{2}$ ) for simplicity.

Once all the distance estimates in (1) are available, the noisy measurements and the NLOS biases at different FTs yield circles which do not intersect at the same point, resulting in the following inconsistent equations

$$
\left(x-x_{i}\right)^{2}+\left(y-y_{i}\right)^{2}=\hat{d}_{i}^{2}, \quad i=1,2, \ldots, N .
$$

A common way to solve for the target node's location from (2) is to perform a non-linear weighted least squares (NL-WLS) estimation [1]; i.e.,

$$
\hat{\mathbf{x}}=\arg \min _{\mathbf{x}}\left\{\sum_{i=1}^{N} \beta_{i}\left(\hat{d}_{i}-\left\|\mathbf{x}-\mathbf{x}_{i}\right\|\right)^{2}\right\}
$$

where the weight $\beta_{i}$ can be chosen to reflect the reliability of the signal received at the $i$ th FT. We may set $\beta_{i}=1 \forall i$ if no information about the reliability of the FTs is available, which yields the NLS solution [11].

As discussed previously, solving for (3) normally requires numerical search techniques such as the steepest descent or the Gauss-Newton techniques. As an alternative to the NL-WLS solution, it is possible to use the techniques proposed in [5], [6] in order to obtain a linear set of equations. By fixing one of the non-linear expressions in (2) for an arbitrary FT (called a reference FT), subtracting it from the rest of the expressions, and after some mathematical manipulation, we may obtain the following linear model

$$
\mathbf{A x}=\mathbf{p}
$$

where, letting the reference FT to be FT- $r$, we have

$$
\begin{gathered}
\mathbf{A}=2\left[\begin{array}{cc}
x_{1}-x_{r} & y_{1}-y_{r} \\
\vdots & \vdots \\
x_{r-1}-x_{r} & y_{r-1}-y_{r} \\
x_{r+1}-x_{r} & y_{r+1}-y_{r} \\
\vdots & \vdots \\
x_{N}-x_{r} & y_{N}-y_{r}
\end{array}\right], \\
\mathbf{p}=\mathbf{p}_{c}+\mathbf{p}_{n},
\end{gathered}
$$

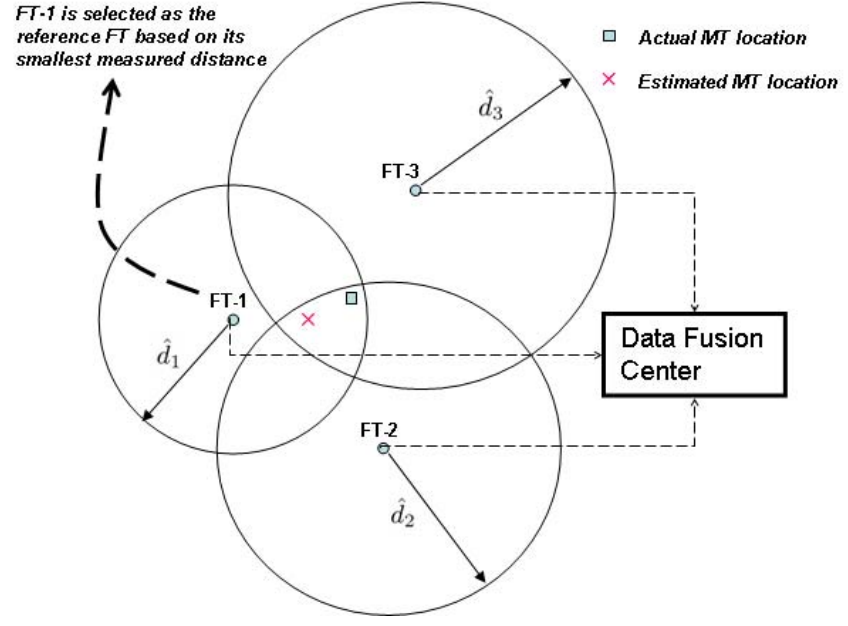

Fig. 1. Illustration of localization problem in a wireless mobile network and selection of the reference FT based on the smallest measured distance.

where the constant and the noisy components of vector $\mathbf{p}$ are denoted by $\mathbf{p}_{c}$ and $\mathbf{p}_{n}$, respectively. From (2), the constant part of $\mathbf{p}$ can be obtained as

$$
\mathbf{p}_{c}=\left[\begin{array}{c}
d_{r}^{2}-d_{1}^{2}-k_{r}+k_{1} \\
\vdots \\
d_{r}^{2}-d_{r-1}^{2}-k_{r}+k_{r-1} \\
d_{r}^{2}-d_{r+1}^{2}-k_{r}+k_{r+1} \\
\vdots \\
d_{r}^{2}-d_{N}^{2}-k_{r}+k_{N}
\end{array}\right],
$$

where $k_{i}=x_{i}^{2}+y_{i}^{2}$.

In order to derive the noisy part of $\mathbf{p}$, we consider the LOS and the NLOS scenarios separately. First, consider that all the FTs are in LOS (i.e., $b_{i}=0 \forall i$ ). Then, we may obtain

$$
\mathbf{p}_{n}=\left[\begin{array}{c}
2 d_{r} n_{r}-2 d_{1} n_{1}+n_{r}^{2}-n_{1}^{2} \\
\vdots \\
2 d_{r} n_{r}-2 d_{r-1} n_{r-1}+n_{r}^{2}-n_{r-1}^{2} \\
2 d_{r} n_{r}-2 d_{r+1} n_{r+1}+n_{r}^{2}-n_{r+1}^{2} \\
\vdots \\
2 d_{r} n_{r}-2 d_{N} n_{N}+n_{r}^{2}-n_{N}^{2}
\end{array}\right] .
$$

In the presence of NLOS bias, the perturbation in the vector $\mathbf{p}$ in (6) will be larger, which will degrade the accuracy compared to LOS scenarios. We may re-write $p$ in NLOS scenarios as

$$
\mathbf{p}=\mathbf{p}_{c}+\tilde{\mathbf{p}}_{n},
$$

where $\mathbf{p}_{c}$ is as in (7) and $\tilde{\mathbf{p}}_{n}$ is given by

$$
\tilde{\mathbf{p}}_{n}=\mathbf{p}_{n}+\tilde{\mathbf{b}} \text {. }
$$


This full text paper was peer reviewed at the direction of IEEE Communications Society subject matter experts for publication in the WCNC 2008 proceedings.

By plugging (1) into (2) and after some manipulation, we may derive the $i$ th term of $\tilde{\mathbf{b}}$ as

$$
[\tilde{\mathbf{b}}]_{i}=b_{r}^{2}-b_{\tilde{i}}^{2}+2\left(d_{r} b_{r}-d_{\tilde{i}} b_{\tilde{i}}+b_{r} n_{r}-b_{\tilde{i}} n_{\tilde{i}}\right),
$$

where

$$
\tilde{i}= \begin{cases}i & , \quad i<r \\ i+1 & , \quad i \geq r .\end{cases}
$$

\section{A. Prior Art LLS Localization Techniques}

Given the linear model in (4), a simple LLS estimator to obtain the MT location is given by [5]

$$
\hat{\mathbf{x}}=\left(\mathbf{A}^{T} \mathbf{A}\right)^{-1} \mathbf{A}^{T} \mathbf{p} \text {. }
$$

In here, we call the estimator in (13) as the LLS-1. Note that $L L S-1$ utilizes the measurements $\hat{d}_{i}, i=1, \ldots, N$, only through the terms $\hat{d}_{r}^{2}-\hat{d}_{i}^{2}$, for $i=1, \ldots, N$ and $i \neq r$. Therefore, the measurement set for $L L S-1$ effectively becomes

$$
\tilde{d}_{i}=\hat{d}_{r}^{2}-\hat{d}_{i}^{2}, \quad i=1, \ldots, N, \quad i \neq r .
$$

Another LLS approach (call it LLS-2) obtains $N \times(N-$ 1)/2 linear equations by subtracting each equation from all of the other equations [6]. In the $L L S-2$ technique, the following observations are employed for position estimation:

$$
\check{d}_{i j}=\hat{d}_{i}^{2}-\hat{d}_{j}^{2}, \quad i, j=1,2, \ldots, N, i<j .
$$

Similar to the $L L S-1$, the linear LS solution as in (13) is obtained for the position of the target node in the LLS-2 technique.

In a third LLS technique (call it $L L S-3$ ), instead of obtaining the difference of the equations directly as in the LLS1 and $L L S-2$ approaches, the average of the measurements is obtained first, and this average is subtracted from all the equations resulting in $N$ linear relations [7]. Then, the linear LS solution as in (13) is obtained for the position of the target node. The observation set employed in the LLS-3 technique can be expressed as

$$
\bar{d}_{i}=\hat{d}_{i}^{2}-\frac{1}{N} \sum_{j=1}^{N} \hat{d}_{j}^{2}, \quad i=1,2, \ldots, N .
$$

\section{REFERENCE FT SELECTION AND MLE FOR LOS AND NLOS SCENARIOS}

\section{A. Reference FT Selection}

The LLS-1 solution in (13) selects an arbitrary FT as the reference FT. However, observing the noisy terms in $\mathbf{p}_{n}$ given in (8), we can see that all the rows of the vector $\mathbf{p}_{n}$ depend on the true distance between the MT and the reference FT. If the FT is away from the MT location, this implies that all the elements of vector $\mathbf{p}$ will be more noisy, degrading the localization accuracy. Hence, how the reference FT is selected may considerably affect the mean square error (MSE) of the estimator. A simple method to select the reference FT for improved location accuracy in LOS scenarios is to choose the FT with the smallest measured distance among all the distance measurements. The index of the reference FT that has the smallest measured distance is given by

$$
r=\arg \min _{i}\left\{\hat{d}_{i}\right\}, \quad i=1,2, \ldots, N .
$$

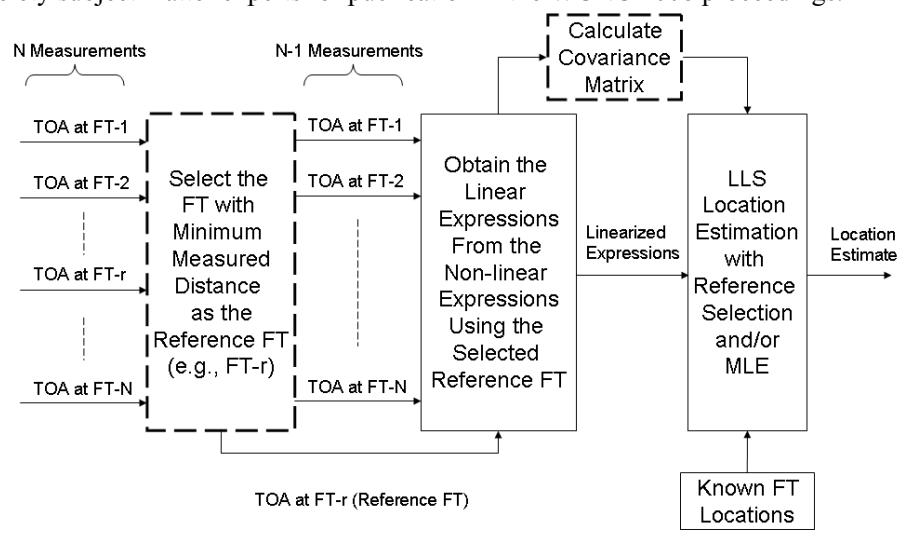

Fig. 2. Block diagram of the proposed reference FT selection and MLE using the covariance matrix. Modifications to a conventional LLS are indicated in dashed boxes.

Then, the matrix $\mathbf{A}$ and the vector $\mathbf{p}$ can be obtained using the selected reference FT (FT- $r$ ), and we refer to the resulting estimator as LLS with reference selection ( $L L S-R S)$. A simple localization scenario where the reference FT is selected based on its minimum measured distance is illustrated in Fig. 1. In this example, FT-1 is used to obtain the linear model from non-linear expressions since $\hat{d}_{1}$ is the minimum among all the measured distances. Note that for a better accuracy, it may also be possible to include the variance of distance measurements as a second criteria while selecting the reference FT. Since we assume that the measured distance variances are the same at different FTs in this paper, we do not consider it here. Nevertheless, in practical scenarios, the variance of the measurements for closer distances between the FT and the MT would likely be smaller, which further motivates the use of (17) for selecting the reference FT. A generic block diagram of $L L S-R S$ is illustrated in Fig. 2 along with the MLE technique to be introduced in the next section.

In NLOS scenarios, how to select the reference FT becomes more complicated. In particular, the $b_{r}^{2}$, the $d_{r} b_{r}$, and the $b_{r} n_{r}$ terms for the reference FT in (11) may dominate, and it may become undesirable to select an NLOS biased FT as a reference FT even for small bias values. Therefore, a simple reference selection technique utilizing the minimum distance measurement criteria and only the LOS FTs can be written as (call it $L L S-R S-N L O S$ )

$$
r=\arg \min _{i}\left\{\hat{d}_{i}\right\}, \quad i \in \mathcal{C}_{L O S},
$$

where $\mathcal{C}_{L O S}$ denotes the index set for all the LOS FTs. Some NLOS identification techniques as in [12], [13] may be used to determine the NLOS FTs and exclude them from the set $\mathcal{C}_{L O S}$. Note that the geometry of the nodes and how the reference FT is placed with respect to the NLOS FT and the MT becomes more important in an NLOS scenario. A drawback of (18) is that it never selects the NLOS FT as a reference. However, for certain cases where NLOS bias is small and when the MT is sufficiently close to the NLOS FT, it may be preferable to select it as the reference.

Another possible approach in NLOS scenarios is, if some estimates $\hat{b}_{i}$ of the NLOS bias are available, we may use $\hat{d}_{i}-$ 
$\hat{b}_{i}$ as the corrected measurements and use the LOS reference selection rule in (17). However, obtaining the estimate $\hat{b}_{i}$ is not typically easy. Nevertheless, in certain cases, it may be possible to know the statistics of $b_{i}$. Letting $\tilde{\mu}_{i}=\mathrm{E}\left\{b_{i}\right\}$ to denote the mean of $b_{i}$, we may use the following decision rule for selecting the reference FT when the bias statistics are available

$$
r=\arg \min _{i}\left\{\hat{d}_{i}-\tilde{\mu}_{i}\right\}, \quad i=1,2, \ldots, N .
$$

\section{B. MLE for LOS Scenarios}

While the reference FT selection discussed above improves the location accuracy, it does not account for the correlation between the rows of the vector $\mathbf{p}_{n}$, which become correlated during the linearization process. To our best knowledge, this correlation was not explicitly considered in the prior art techniques on LLS, which we will use below for improving the location accuracy. As discussed in [14], the optimum estimator in the presence of correlated observations is given by an ML estimator. First, we consider the following modification of the relationship in (6) for a LOS scenario

$$
\mathbf{p}=\mathbf{A} \tilde{\mathbf{x}}+\mathbf{p}_{n}
$$

where $\tilde{\mathbf{x}}$ is the actual location of the MT, and hence $\mathbf{p}_{c}=\mathbf{A} \tilde{\mathbf{x}}$. Then, based on (20), the MLE ${ }^{1}$ for this linear model can be written as [14]

$$
\hat{\mathbf{x}}=\left(\mathbf{A}^{T} \mathbf{C}^{-1} \mathbf{A}\right)^{-1} \mathbf{A}^{T} \mathbf{C}^{-1} \mathbf{p}
$$

where $\mathbf{C}=\operatorname{Cov}\left(\mathbf{p}_{n}\right)$ is the covariance matrix of vector $\mathbf{p}_{n}$.

When all the FTs are in LOS, the covariance matrix of vector $\mathbf{p}_{n}$ can be derived as (see Appendix A)

$$
\begin{aligned}
\mathbf{C}=4 d_{r}^{2} \sigma^{2}+2 \sigma^{4}+\operatorname{diag}\left\{4 \sigma^{2} d_{1}^{2}+2 \sigma^{4}, \ldots,\right. \\
\left.4 \sigma^{2} d_{i}^{2}+2 \sigma^{4}, \ldots, 4 \sigma^{2} d_{N}^{2}+2 \sigma^{4}\right\},
\end{aligned}
$$

with $i \in\{1,2, \ldots, N\}, i \neq r$, and where $\operatorname{diag}\left\{\lambda_{1}, \ldots, \lambda_{N}\right\}$ is a diagonal matrix obtained by placing $\lambda_{i}$ on the diagonal of an $(N-1) \times(N-1)$ zero matrix $\forall i$. Note that since $d_{i}$ are not available in practice, the noisy measurements $\hat{d}_{i}$ can be used to evaluate the covariance matrix. Once an estimate of $\mathbf{x}$ is available, then, its PDF can be shown to be [14]

$$
\hat{\mathbf{x}} \sim \mathcal{N}\left(\tilde{\mathbf{x}},\left(\mathbf{A}^{T} \mathbf{C}^{-1} \mathbf{A}\right)^{-1}\right) .
$$

\section{MLE for NLOS Scenarios}

Now consider that some of the FTs are in NLOS with the MT. Similar to as discussed in previous section, if some estimate $\hat{b}_{i}$ of the NLOS bias are available, we may correct the distance measurements as $\hat{d}_{i}-\hat{b}_{i}$, and then apply the MLE in (21). While this may not be very practical, we may have some prior knowledge about the statistics of NLOS bias. In here, we consider that the NLOS bias is a Gaussian distributed random variable as in [8], where $b_{i} \sim \mathcal{N}\left(\tilde{\mu}_{i}, \tilde{\sigma}_{i}^{2}\right)$ for some

\footnotetext{
${ }^{1}$ Note that in order have the MLE as in (21), the elements of $\mathbf{p}_{n}$ should be zero-mean and Gaussian distributed random variables. While there are some non-Gaussian terms (i.e., the noise-square terms) in $\mathbf{p}_{n}$, they are assumed to be negligible, or fit closely to a Gaussian distribution to obtain the MLE.
}

NLOS FTs. If such information is available, we may modify the distance measurements as

$$
\tilde{d}_{i}=\hat{d}_{i}-\tilde{\mu}_{i}=d_{i}+\tilde{n}_{i}
$$

where $\tilde{n}_{i} \sim \mathcal{N}\left(0, \sigma^{2}+\tilde{\sigma}_{i}^{2}\right)$. Then, the covariance matrix of $\mathbf{p}_{n}$ using (24) as distance measurements can be written as (see Appendix A)

$\mathbf{C}=4 d_{r}^{2}\left(\sigma^{2}+\tilde{\sigma}_{r}^{2}\right)+2\left(\sigma^{2}+\tilde{\sigma}_{r}^{2}\right)^{2}+\operatorname{diag}\left\{\gamma_{1}, \ldots, \gamma_{i}, \ldots, \gamma_{N}\right\}$,

with $i \in\{1,2, \ldots, N\}, i \neq r$, and where $\gamma_{i}=4 d_{i}^{2}\left(\sigma^{2}+\tilde{\sigma}_{i}^{2}\right)+$ $2\left(\sigma^{2}+\tilde{\sigma}_{i}^{2}\right)^{2}$. Once we obtain (25), we may plug it into (21) to obtain the MLE solution.

\section{Simulation Results}

Monte-Carlo simulations are performed in order to compare the proposed LLS estimators with the prior art techniques. Four FTs are positioned on the corners of a square at $\mathbf{x}_{1}=$ $[-50,-50], \mathbf{x}_{2}=[50,-50], \mathbf{x}_{3}=[-50,50]$, and $\mathbf{x}_{4}=$ $[50,50]$ (all in meters). For LLS-1, the FT-1 is used as a reference FT. The MT location $\tilde{\mathbf{x}}$ is changed with 15 meter intervals within $[-45,45] \mathrm{m}$ both in $x$ and $y$ directions, yielding a $7 \times 7$ grid of possible MT locations. The MSE of different techniques are simulated at each location on the grid, and then averaged over all the MT locations on the grid.

The LOS results in Fig. 3 show that the LLS-1 performs worst compared to all the other techniques. The $L L S-2$ and $L L S-3$ techniques perform slightly better than $L L S-1$, and their MSEs are identical. However, they are both beaten by the LLS-RS technique. The accuracy of the MLE solution is not affected from how the reference FT is selected, and it performs slightly better than that of $L L S-R S$. The accuracy gains using the LLS-RS and the MLE improve with increasing noise variance (i.e., decreasing signal-to-noise ratio). The CRLBs provide the lower bound for all the other techniques.

For NLOS simulations, we consider that FT-4 is the NLOS FT, and the rest of the FTs are all in LOS. In Fig. 4, simulation results for $L L S-1$ when different FTs are selected as the reference FT are presented for different noise variances $\left(\sigma^{2} \in\{0.3,1,3\} \mathrm{m}^{2}\right)$. The NLOS bias at FT-4 is changed from 0 meters to 3 meters. The CRLBs with biased measurements are also indicated. A critical observation is that when FT-4 is selected as the reference FT, the MSE is the worst for all scenarios. This verifies the claim in Section III-A that on the average, an NLOS FT should not be selected as a reference $\mathrm{FT}^{2}$. On the other hand, FT-1, which is the FT that is furthest from the NLOS FT, is always the best reference FT to select.

The NLOS simulations in Figs. 5-7 compare the accuracies of the proposed techniques with those of $L L S-1$ for different values of $\sigma^{2}$. The FT-1 (which shows to be the best reference in Fig. 4) is always selected as the reference FT for $L L S-1$. The results show that the LLS-RS and the LLS-RS-NLOS perform better than LLS-1 for LOS scenarios or for small NLOS bias values. When the NLOS bias value gets larger,

\footnotetext{
${ }^{2}$ Note again that the MSE is averaged over different locations on the grid, and given $b_{4}$, there may be individual locations close to FT-4 on the grid where selecting FT-4 as a reference may be preferable.
} 
This full text paper was peer reviewed at the direction of IEEE Communications Society subject matter experts for publication in the WCNC 2008 proceedings.

after some point, the LLS-1 starts performing better. This is because the $L L S-R S$ uses the measured distances $\hat{d}_{i}$, which gets considerably biased as the NLOS bias increases. For larger $\sigma^{2}$, the range of NLOS bias values where the LLS-RS beats the $L L S-1$ is larger. Moreover, we observe that there is only marginal improvement of using LLS-RS-NLOS rather than $L L S-R S$, which appears when $\sigma^{2}$ is small and when NLOS bias is large. This is because LLS-RS-NLOS never selects the NLOS FT as a reference FT even when MT is very close to it. In all the three figures, MLE with perfect bias knowledge performs close to the CRLB (as in a LOS scenario) $)^{3}$, while without any knowledge of $b_{i}$, it still performs better than those of $L L S-R S$.

Finally in Fig. 8, performances of different techniques when $b_{4} \sim \mathcal{N}(1,0.2)$ are compared. Due to simulation time constraints, only a single grid location at $\tilde{x}=[10,10]$ meters is considered. The results show that when some statistics about the NLOS bias are available, the localization accuracy of the LLS-RS and MLE can be improved by using corrected measurements. The MLE with know bias statistics is very close to the CRLB even in the NLOS scenario. On the other hand, when there is no a-priori information available about the NLOS bias, both the LLS-RS and MLE is beaten by LLS-1 at small $\sigma^{2}$, as also implied by Figs. 5-7.

\section{CONCLUSION}

In this paper, various algorithms have been proposed for the enhancement of the LLS localization techniques and performance comparisons have been presented for LOS and NLOS scenarios. In the first technique ( $L L S-R S$ ), the FT with the smallest distance measurement is selected as the reference FT. The second approach (MLE) uses the correlation of the observations to obtain a more accurate estimator. Simulation results show that both techniques perform better than the other prior art techniques (e.g., $L L S-1, L L S-2$, and $L L S$-3) in LOS scenarios. In NLOS scenarios, they are more effective at low SNRs and small NLOS bias values. If some prior statistics about the NLOS bias are available, the accuracies of both techniques can be improved. Our future work includes detailed theoretical analysis of the proposed techniques in NLOS scenarios.

\section{APPENDIX}

\section{A. Derivation of the Covariance Matrices}

The elements of the covariance matrix of a vector $\mathbf{p}_{n}$ is calculated as

$$
[\mathbf{C}]_{i j}=\mathrm{E}\left\{\left(\left[\mathbf{p}_{n}\right]_{i}-\mathrm{E}\left\{\left[\mathbf{p}_{n}\right]_{i}\right\}\right)\left(\left[\mathbf{p}_{n}\right]_{j}-\mathrm{E}\left\{\left[\mathbf{p}_{n}\right]_{j}\right\}\right)\right\}
$$

where $\mathrm{E}\left\{\mathbf{p}_{n}\right\}$ denotes the expectation of $\mathbf{p}_{n}$. Then, in LOS scenarios, the covariance matrix can be derived as

$$
\begin{aligned}
{[\mathbf{C}]_{i j}=} & \mathrm{E}\left\{\left(2 d_{r} n_{r}-2 d_{i} n_{i}+n_{r}^{2}-n_{i}^{2}\right)\right. \\
& \left.\times\left(2 d_{r} n_{r}-2 d_{j} n_{j}+n_{r}^{2}-n_{j}^{2}\right)\right\} \\
= & 4 d_{r}^{2} \sigma^{2}+2 \sigma^{4}+I(i, j)\left(4 d_{i}^{2} \sigma^{2}+2 \sigma^{4}\right),
\end{aligned}
$$

\footnotetext{
${ }^{3}$ Note that we may also consider LLS-RS with perfect bias knowledge,
} which would yield the same accuracy as in a LOS scenario. where $I(i, j)$ is an indicator function which is 1 for $i=j$, and is 0 otherwise. From (27), we can easily obtain (22).

In an NLOS scenario, when only the statistics of the NLOS bias are available, similar to (27), the covariance matrix can be written as

$$
\begin{aligned}
{[\mathbf{C}]_{i j}=\mathrm{E} } & \left\{\left(2 d_{r} \tilde{n}_{r}-2 d_{i} \tilde{n}_{i}+\tilde{n}_{r}^{2}-\tilde{n}_{i}^{2}\right)\right. \\
& \left.\times\left(2 d_{r} \tilde{n}_{r}-2 d_{j} \tilde{n}_{j}+\tilde{n}_{r}^{2}-\tilde{n}_{j}^{2}\right)\right\} \\
=4 & d_{r}^{2}\left(\sigma^{2}+\tilde{\sigma}_{i}^{2}\right)+2\left(\sigma^{2}+\tilde{\sigma}_{i}^{2}\right)+ \\
& I(i, j)\left(4 d_{i}^{2}\left(\sigma^{2}+\tilde{\sigma}_{i}^{2}\right)+2\left(\sigma^{2}+\tilde{\sigma}_{i}^{2}\right)\right) .
\end{aligned}
$$

Then, (25) follows easily from (28).

\section{REFERENCES}

[1] J. J. Caffery and G. L. Stuber, "Overview of radiolocation in CDMA cellular systems," IEEE Commun. Mag., vol. 36, no. 4, pp. 38-45, Apr. 1998.

[2] S. Gezici, Z. Tian, G. B. Giannakis, H. Kobayashi, A. F. Molisch, H. V. Poor, and Z. Sahinoglu, "Localization via ultra-wideband radios: a look at positioning aspects for future sensor networks," IEEE Signal Processing Mag., vol. 22, no. 4, pp. 70-84, July 2005.

[3] F. Gustafsson and F. Gunnarsson, "Mobile positioning using wireless networks: Possibilites and fundamental limitations based on available wireless network measurements," IEEE Sig. Proc. Mag., vol. 22, no. 4, pp. 41-53, July 2005.

[4] W. Kim, J. G. Lee, and G. I. Jee, "The interior-point method for an optimal treatment of bias in trilateration location," IEEE Trans. Vehic. Technol., vol. 55, no. 4, pp. 1291-1301, July 2006.

[5] J. J. Caffery, "A new approach to the geometry of TOA location," in Proc. IEEE Vehic. Technol. Conf. (VTC), vol. 4, Boston, MA, Sep. 2000, pp. 1943-1949.

[6] S. Venkatesh and R. M. Buehrer, "A linear programming approach to NLOS error mitigation in sensor networks," in Proc. IEEE Int. Conf. on Information Processing in Sensor Networks (IPSN), Nashville, TN, Apr. 2006, pp. 301-308.

[7] Z. Li, W. Trappe, Y. Zhang, and B. Nath, "Robust statistical methods for securing wireless localization in sensor networks," in Proc. IEEE Int. Symp. Information Processing in Sensor Networks (IPSN), Los Angeles, CA, Apr. 2005, pp. 91-98.

[8] V. Dizdarevic and K. Witrisal, "On impact of topology and cost function on LSE position determination in wireless networks," in Proc. Workshop on Positioning, Navigation, and Commun. (WPNC), Hannover, Germany, Mar. 2006, pp. 129-138.

[9] I. Guvenc, C. C. Chong, and F. Watanabe, "Analysis of a linear leastsquares localization technique in LOS and NLOS environments," in Proc. IEEE Vehic. Technol. Conf. (VTC), Dublin, Ireland, Apr. 2007, pp. 1886-1890.

[10] Y. T. Chan, H. Y. C. Hang, and P. C. Ching, "Exact and approximate maximum likelihood localization algorithms," IEEE Trans. Vehicular Technology, vol. 55, no. 1, pp. 10-16, Jan. 2006.

[11] S. Gezici, "A survey on wireless position estimation," Springer Wireless Personal Communications, Special Issue Towards Global and Seamless Personal Navigation, Oct. 2007.

[12] S. Gezici, H. Kobayashi, and H. V. Poor, "Non-parametric non-line-ofsight identification," in Proc. IEEE Vehic. Technol. Conf. (VTC), vol. 4, Orlando, FL, Oct. 2003, pp. 2544-2548.

[13] I. Guvenc, C. C. Chong, and F. Watanabe, "NLOS identification and mitigation for UWB localization systems," in Proc. IEEE Int. Conf. Wireless Commun. Networking (WCNC), Hong Kong, Mar. 2007, pp. 1571-1576.

[14] S. M. Kay, Fundamentals of Statistical Signal Processing: Estimation Theory. Upper Saddle River, NJ: Prentice Hall, Inc., 1993. 


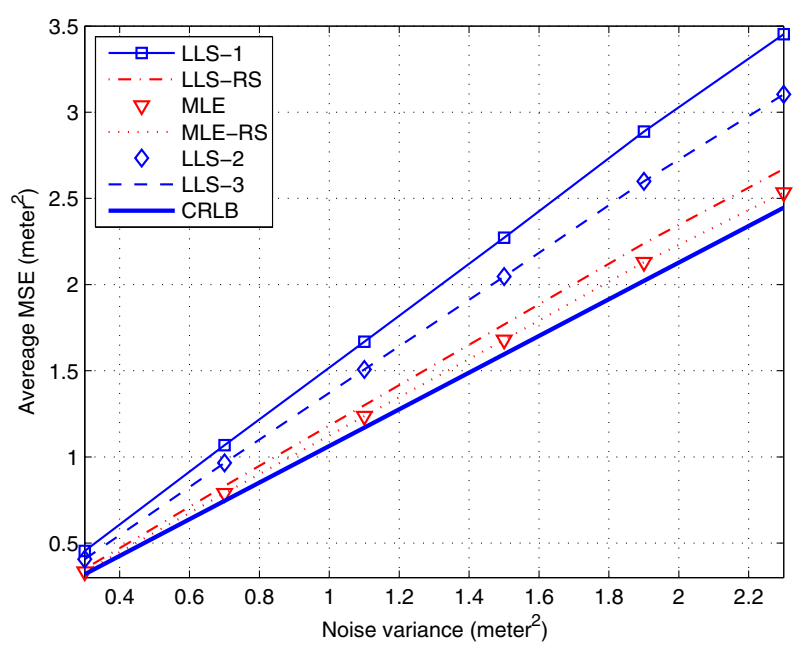

Fig. 3. Comparison of different techniques in LOS scenario.

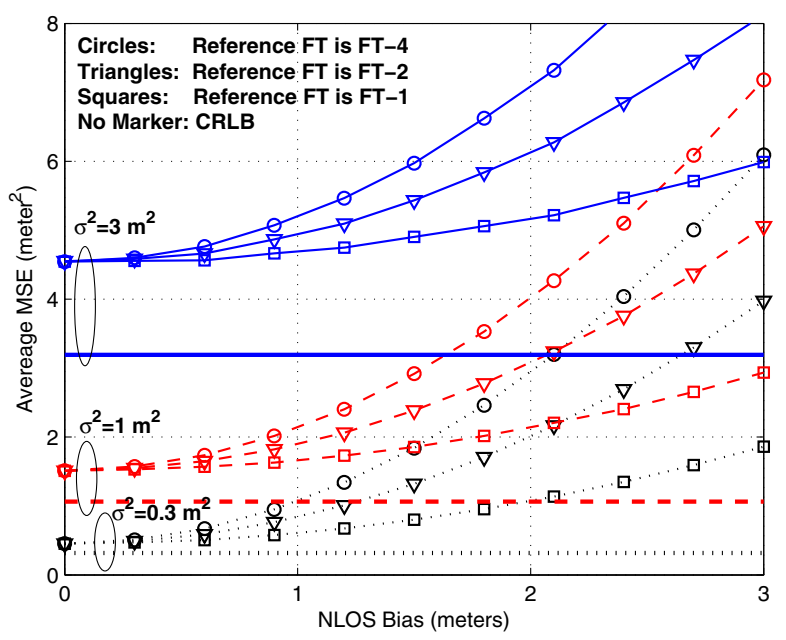

Fig. 4. Comparison of LLS-1 for different NLOS scenarios.

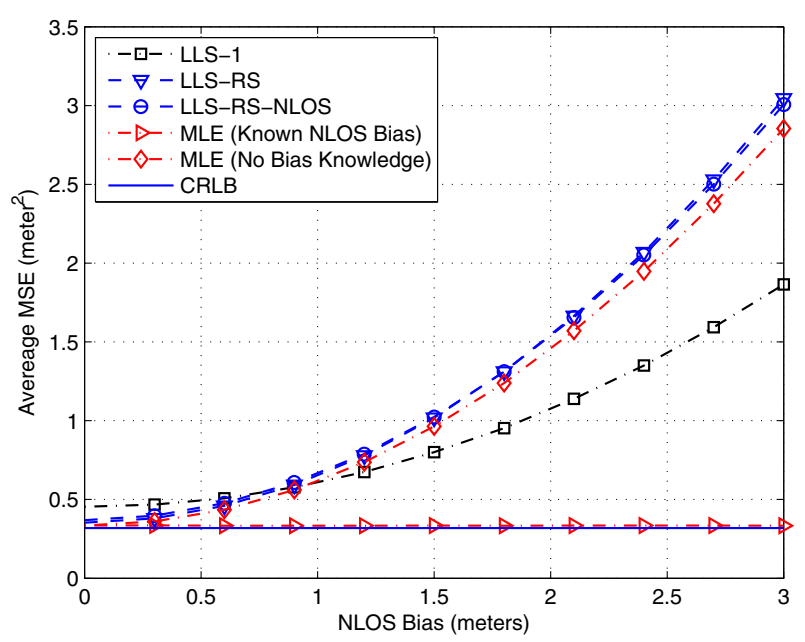

Fig. 5. MSE with respect to NLOS bias $\left(\sigma^{2}=0.3 \mathrm{~m}^{2}\right)$.

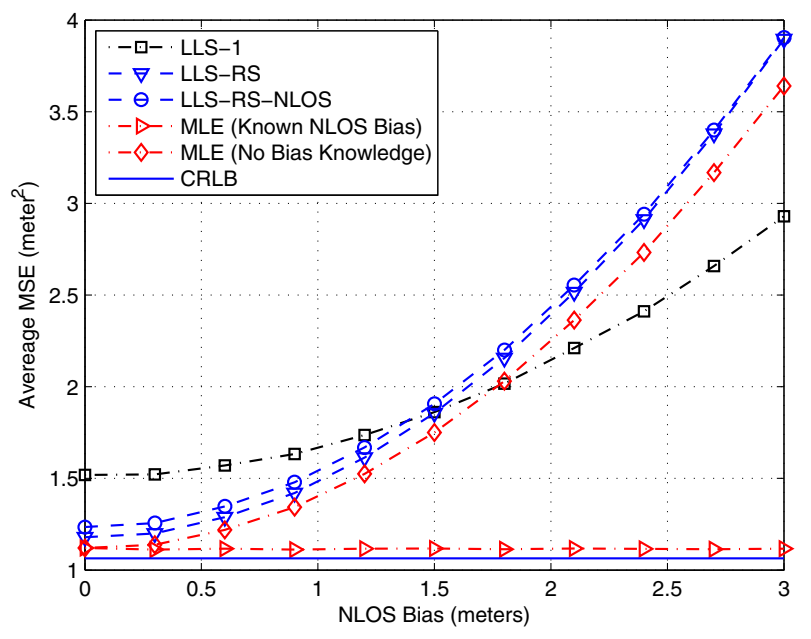

Fig. 6. MSE with respect to NLOS bias $\left(\sigma^{2}=1 \mathrm{~m}^{2}\right)$.

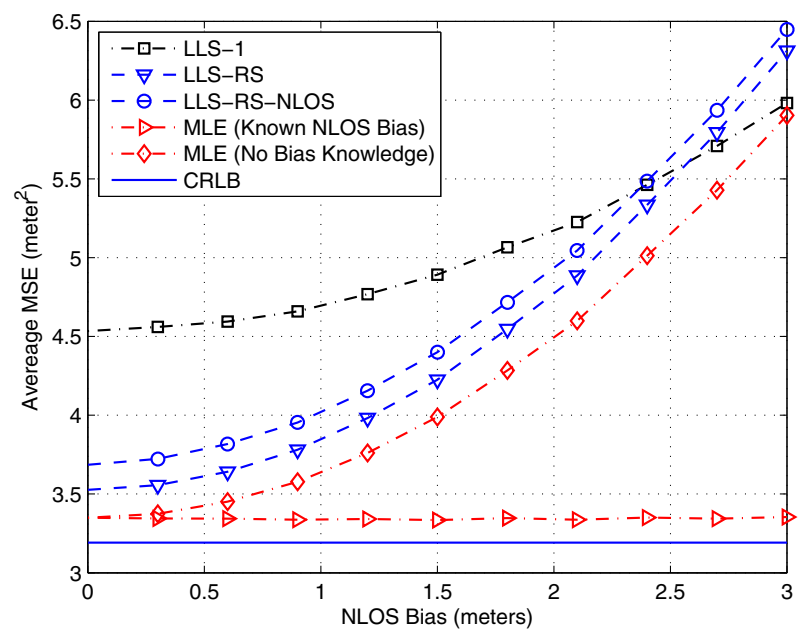

Fig. 7. MSE with respect to NLOS bias $\left(\sigma^{2}=3 \mathrm{~m}^{2}\right)$.

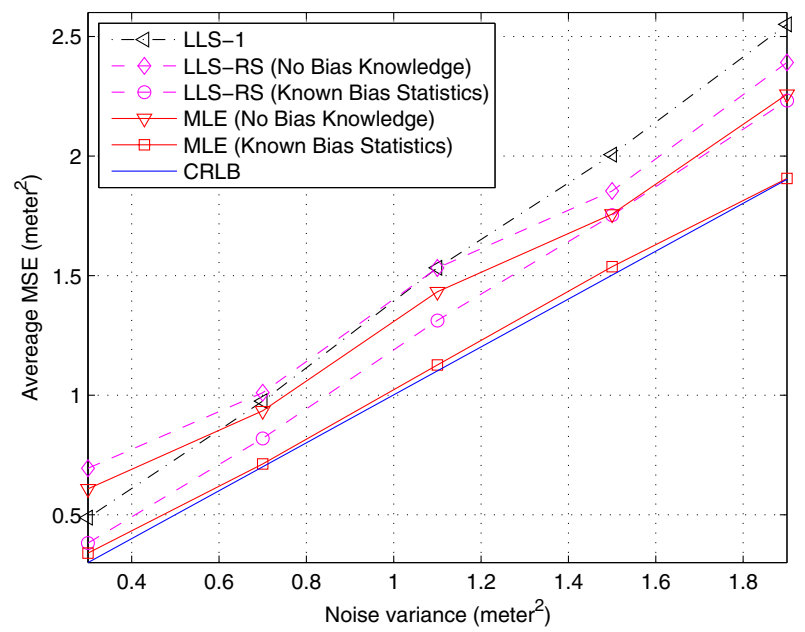

Fig. 8. Comparison of different techniques when the NLOS bias is Gaussian distributed. 